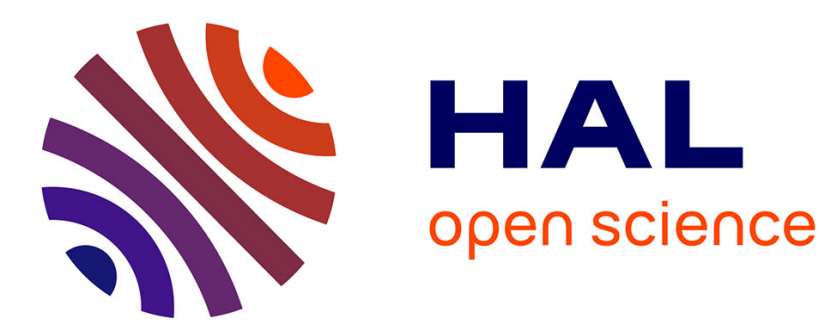

\title{
Dire et faire le commun
}

Claire Judde de Larivière, Julien Weisbein

\section{To cite this version:}

Claire Judde de Larivière, Julien Weisbein. Dire et faire le commun: Les formes de la politisation ordinaire du Moyen Âge à nos jours. Politix, 2017, n 119 (119), pp.9-30. 10.3917/pox.119.0007 . hal-02025803

\section{HAL Id: hal-02025803 https://hal.science/hal-02025803}

Submitted on 10 Sep 2021

HAL is a multi-disciplinary open access archive for the deposit and dissemination of scientific research documents, whether they are published or not. The documents may come from teaching and research institutions in France or abroad, or from public or private research centers.
L'archive ouverte pluridisciplinaire HAL, est destinée au dépôt et à la diffusion de documents scientifiques de niveau recherche, publiés ou non, émanant des établissements d'enseignement et de recherche français ou étrangers, des laboratoires publics ou privés. 


\section{DIRE ET FAIRE LE COMMUN}

Les formes de la politisation ordinaire du Moyen Âge à nos jours

Claire Judde de Larivière, Julien Weisbein

De Boeck Supérieur | « Politix »

$2017 / 3 n^{\circ} 119 \mid$ pages 7 à 30

ISSN 0295-2319

ISBN 9782807391253

Article disponible en ligne à l'adresse :

https://www.cairn.info/revue-politix-2017-3-page-7.htm

\section{Pour citer cet article :}

Claire Judde de Larivière, Julien Weisbein « Dire et faire le commun. Les formes de la politisation ordinaire du Moyen Âge à nos jours », Politix 2017/3 ( $\left.\mathrm{n}^{\circ} 119\right)$, p. 7-30. DOI 10.3917/pox.119.0007

Distribution électronique Cairn.info pour De Boeck Supérieur.

(C) De Boeck Supérieur. Tous droits réservés pour tous pays.

La reproduction ou représentation de cet article, notamment par photocopie, n'est autorisée que dans les limites des conditions générales d'utilisation du site ou, le cas échéant, des conditions générales de la licence souscrite par votre établissement. Toute autre reproduction ou représentation, en tout ou partie, sous quelque forme et de quelque manière que ce soit, est interdite sauf accord préalable et écrit de l'éditeur, en dehors des cas prévus par la législation en vigueur en France. Il est précisé que son stockage dans une base de données est également interdit. 


\section{Dossier}

Politiques du commun (XVI ${ }^{\mathrm{e}}$-XIX ${ }^{\mathrm{e}}$ siècles)

Coordonné par Claire Judde de Larivière 


\title{
Dire et faire le commun
}

\section{Les formes de la politisation ordinaire du Moyen Âge à nos jours}

\author{
Claire Judde de LaRIvière et Julien Weisbein
}

\begin{abstract}
Résumé - Cet article propose un cadrage historiographique et conceptuel de la question des pratiques ordinaires du politique et de leur centralité dans l'élaboration du commun, en mettant en perspective les travaux des historien.ne.s et ceux des politistes. Suivant des parcours distincts, les deux disciplines ont élaboré sur cette question des objets qui semblent aujourd'hui converger sans que les connexions soient encore faites de façon formelle. Partant de ces constats et de l'analyse de travaux récents, cet article propose des définitions et des principes d'étude de ces pratiques politiques ordinaires, et interroge les sources à notre disposition, les formes d'action et de discours qu'elles révèlent, le contenu et le sens de ces pratiques, ainsi que leur influence sur la construction de l'ordre politique tel que l'élabore ensuite la puissance publique. Cette introduction reflète les attendus théoriques ayant présidé à l'élaboration des articles du dossier, et questionne notamment les définitions de l'ordinaire, les notions de commun, d'ancrage en proximité ou de conjonctures fluides qui ont structuré notre réflexion collective.
\end{abstract}

Mots clés - commun, politisation, peuple, ordinaire, histoire 
$\mathrm{E}$ t si le discrédit actuel des formats politiques conventionnels et l'inflation concomitante d' « innovations démocratiques » invitaient à rappeler qu'il existe d'autres formes possibles de politique ${ }^{1}$ ? Derrière cette question se trouvent aujourd'hui rassemblés de nombreux objets des sciences sociales du politique auxquels l'éclairage diachronique ici apporté voudrait contribuer : la politisation, la compétence politique, les rapports ordinaires au politique, la citoyenneté, la délibération, les formes de résistance à la domination, l'encastrement social du politique et au final, plus fondamentalement, l'autonomie relative du champ politique.

Voilà le pari de ce dossier : au-delà d'une définition canonique et professionnalisée du politique, telle que la tradition wébérienne nous l'a léguée, et en dépassant la rupture chronologique du XIX ${ }^{\mathrm{e}}$ siècle comme seuil de professionnalisation politique, il s'agit d'étudier en soi et pour soi des formes non spécialisées de politique, c'est-à-dire la façon dont «le politique » (gardons-nous à ce stade d'une définition même sommaire de ce terme) est enchâssé, encastré, reflété, diffusé dans des pratiques sociales ou des grammaires ne relevant pas d'un espace a priori défini comme tel. Pour ce faire, nous proposons un double déplacement : de la politisation - notion qui depuis l'ouvrage dirigé par Jacques Lagroye en $2003^{2}$ cristallise sans doute le plus ce programme de recherche - vers les politisations ordinaires; et, dans une perspective diachronique, à rebours, de l'époque contemporaine vers des périodes plus reculées.

Car pour penser et comprendre la politique au Moyen Âge et à l'époque moderne - mais cela est aussi vrai pour la période contemporaine -, il est essentiel de réintégrer une multitude de pratiques et de formes, d'actions et de discours qualifiés ici d' " ordinaires », et qui ont conduit à la construction d'un domaine politique lié, mais non réductible, à celui de l'État. Sans nier le caractère profondément inégalitaire des sociétés d'Ancien Régime ou d'aujourd'hui, et la force des structures de domination qui s'y exercent, nous voyons dans ces formes alternatives du domaine politique davantage que des reflets imparfaits de formes dominantes, qui fonctionneraient selon des logiques mimétiques ou dans des rapports de force qui leur seraient totalement défavorables.

Il ne s'agira pas pour autant, dans une perspective téléologique, de chercher les linéaments d'une institutionnalisation et d'une professionnalisation du politique. Notre démarche ne se veut ni généalogique ni évolutive. Nous ne tracerons pas une histoire des pratiques politiques ordinaires dont le destin aurait

1. Ce dossier a bénéficié du soutien et a été réalisé dans le cadre du Labex Structurations des Mondes Sociaux (SMS, ANR-11-LABX-0066). Qu'il nous soit permis de remercier ici l'ensemble des contributeurs de ce dossier pour leurs remarques sur cette introduction, les deux évaluateurs anonymes qui ont considérablement amélioré cette dernière, et Quentin Deluermoz pour son soutien et sa contribution décisive tout au long de ce projet, du séminaire à l'origine de ce dossier jusqu'à sa publication dans le présent numéro.

2. Lagroye (J.), dir., La politisation, Paris, Belin, 2003. 
été de devenir, à terme, des pratiques institutionnalisées et professionnalisées. Nous entendons plutôt historiciser l'ordinaire, c'est-à-dire analyser comment celui-ci permet d'envisager d'autres formats du politique avant le XIX ${ }^{e}$ siècle et d'éclairer l'histoire du Moyen Âge et de l'Ancien Régime de façon renouvelée. Mais ce détour par le passé voudrait aussi être un moyen de reconsidérer des phénomènes et des configurations politiques contemporains, en insistant sur les formes alternatives qui s'énoncent sans le prisme d'un espace social et politique spécialisé et qui ne seraient dès lors plus rabattues sur le registre du manque ou de l'incomplétude.

C'est en analysant la façon dont les acteurs mettent en ouvre des actions et des discours s'appuyant sur la défense du commun que nous tâcherons de saisir ces formes oubliées du politique. Car dans les sociétés d'Ancien Régime, et quelle que soit l'échelle d'observation et d'analyse choisie, les sources font émerger une rhétorique de la défense du commun ainsi que des actions visant explicitement à protéger ou atteindre des biens, des valeurs, des principes, relevant du commun. Dire et faire le commun, c'est énoncer une intention politique et lui donner matière. Pour les hommes et les femmes « ordinaires » - en l'occurrence, à travers ce dossier, les sujets du royaume de France divisés par les guerres de Religion, les membres des communautés villageoises de Navarre et les sujets fiscaux de l'Empire germanique au XVIII ${ }^{e}$ siècle, les paroissiens vendéens aux temps de la Révolution française, et les mineurs d'Anzin au XIX ${ }^{e}$ siècle - des moments d'action et d'élocution situés deviennent ainsi des opportunités de mettre en œuvre des pratiques et des discours qui relèvent largement d'une élaboration politique, et pas seulement au sens où ceux-ci provoquent une réaction du pouvoir officiel, mais bien dans leurs intentions propres et dans les actions mises en œuvre.

\section{L'ordinaire et l'hypothèse continuiste}

Du couplage à «l'extraordinaire » qu'implique l'emploi de cette catégorie de l'« ordinaire » émerge d'emblée un problème méthodologique pour l'historien.ne. Car, par leur nature même, les sources de la période antérieure au $\mathrm{XIX}^{\mathrm{e}}$ siècle nous contraignent fréquemment à envisager des contextes " extraordinaires » et de tension politique ${ }^{3}$. Ce sont en effet les conflits juridiques, les grèves, les périodes de révolte, de résistance et de contestation politique, ou les guerres civiles qui ont laissé le plus de traces dans les archives. L'hypothèse partagée par les contributeurs de ce dossier est qu’il faut néanmoins regarder au-delà des configurations violentes et conflictuelles qui saturent les sources,

\footnotetext{
3. Selon Pierre Favre, on ne peut d'ailleurs pas penser l'ordinaire en politique sans prendre en compte un arrière-fond qui renvoie à des situations extraordinaires de coercition : Favre (P.), "Y a-t-il un rapport "ordinaire" au politique? ", in Marie (J.-L.), Dujardin (P.), Balme (R.), dir., L'ordinaire. Modes d'accès et pertinence pour les sciences sociales et humaines, Paris, L'Harmattan, 2002.
} 
au-delà des actions de destruction ou de résistance à l'ordre, pour faire émerger des formes sous-jacentes et omniprésentes, routinières et ordinaires, qui participent à la construction des mondes sociaux. La perturbation et le dysfonctionnement du jeu routinier d'un espace politique sont trop souvent perçus comme les principes de changement d'institutions ou de pouvoirs. Les pratiques ordinaires s'inscrivent en réalité dans une continuité, pour reprendre les termes de Michel Dobry, et nous les replacerons dans une temporalité longue, en pensant avant, pendant et après la crise politique, pour comprendre leur émergence et la façon dont elles se sédimentent. L'hypothèse continuiste nous permettra ainsi de nous focaliser sur les processus de politisation sous-jacents, plutôt que sur les actions éclatantes et violentes qui apparaissent à la surface des sources ${ }^{4}$. En insistant sur la continuité entre temps chauds et froids de l'action, c'est-à-dire entre les crises politiques et les moments de concorde ou de routine où s'élabore le politique, nous analyserons la façon dont les gens ordinaires construisent le monde et le commun, dans différentes situations et configurations historiques.

Comment révéler ce qu'il peut y avoir de politique dans cet état ordinaire des mondes sociaux ? Il existe à ce sujet, dans une grande partie de la littérature, une sorte de dilemme intimidant : à moins de s'abîmer dans une sorte de folklore et de recherche du pittoresque, détecter des formes ordinaires et non spécialisées de politique, c'est-à-dire des politisations détachées des institutions formelles du politique, impliquerait nécessairement un détour philosophique visant à conceptualiser différemment le politique. Celui-ci ne serait dès lors plus attaché à l'exercice d'une domination induite par la division sociale des tâches et à la régulation des différends qu'elle ouvre, mais renverrait plus fondamentalement à la prise en charge de l'historicité des sociétés, à la façon dont elles se pensent, se signifient et s'auto-instituent ${ }^{5}$. Il ne s'agit pas seulement pour nous de produire une nouvelle définition " $\mathrm{du}$ » politique, qui ne serait plus une fonction mais une sorte d'essence, mais surtout de rechercher ces formes alternatives (et empiriquement identifiables) de politisation ailleurs ou avant. Ailleurs, en les étudiant dans des sociétés où la spécialisation du politique est réputée moins forte ${ }^{6}$. Et avant, comme c'est le cas du présent dossier, c'est-àdire dans des configurations historiques antérieures à ce qui est canoniquement défini par l'histoire contemporaine et la science politique comme le moment de la construction et de l'autonomisation du champ politique, qui se seraient réalisées entre les XVIII et ${ }^{e} \mathrm{XIX}^{e}$ siècles.

4. Dobry (M.), Sociologie des crises politiques, Paris, Presses de Sciences Po, 2009 [1986].

5. Voir par exemple Rosanvallon (P.), Pour une histoire conceptuelle du politique, Paris, Seuil, 2003.

6. Scott (J.), La domination et les arts de la résistance. Fragments du discours subalterne, Paris, Éditions Amsterdam, 2009 ; Bayart (J.-F.), Mbembe (A.), Toulabor (C.), Le politique par le bas en Afrique noire. Contributions à une problématique de la démocratie, Paris, Karthala, 1992; Goirand (C.), La politique des favelas, Paris, Karthala, 2000. Sur ces " postérités exotiques » de la définition lagroyenne de la politisation, cf. Aït-Aoudia (M.), Bennani-Chraïbi (M.), Contamin (J.-G.), «Contribution à une histoire sociale de la conception lagroyenne de la politisation ", Critique internationale, 3(48), 2010. 


\section{Science politique et histoire : des échanges à la co-construction}

Parce qu'elle s'aventure sur des périodes où ses concepts semblent anachroniques, ou en tout cas bien moins affirmés, la science politique a, plus que jamais, besoin de l'histoire, comme cette dernière ne peut faire l'économie des questionnements récents développés par la première ${ }^{7}$. Tel est le second pari de ce dossier, qui s'est construit autour d'une forme de collaboration spécifique entre les deux disciplines.

Plusieurs modalités de mise en relation entre politistes et historien.ne.s peuvent être identifiées - une fois dépassée l'indifférence mutuelle, sans doute et malheureusement la plus pratiquée. En premier lieu vient une division sommaire des tâches, héritée de Durkheim : la sociologie politique théorise et l'histoire apporte des exemples du passé pour renforcer la pertinence de la théorie. Dans ce cas, l'histoire est une simple auxiliaire (du point de vue du ou de la politiste) et la science politique est un recueil de schémas à plaquer sur le vivant de l'histoire (du point de vue de l'historien.ne). C'est en second lieu par l'emprunt et l'échange d'objets que les disciplines ont collaboré, comme c'est le cas de la socio-histoire du politique, qui renvoie à l'investissement réalisé par des politistes de terrains passés appartenant jusqu'alors aux historien.ne. $s^{8}$, davantage sans doute qu'à un échange raisonné et surtout équilibré entre les deux communautés savantes ${ }^{9}$. Les échanges de concepts qui, en troisième lieu, voient certaines notions et problématiques circuler entre les deux disciplines, semblent à cet égard plus fréquents. C'est par exemple le cas du dossier de Participations consacré à « La participation au prisme de l'histoire », où l'enquête historique a pour fonction de contrôler la présomption de nouveauté (voire d'innovation) portée par certains discours sur la démocratie délibérative ${ }^{10}$. En ce sens, le dossier « Protagonisme et crises politiques » publié dans Politix en 2015 parvient à renverser la division traditionnelle du travail intellectuel entre histoire et science politique par l'injection dans des réalités contemporaines relevant de travaux de politistes d'un concept forgé par un historien (le protagonisme de Haim Burstin) pour un objet historique particulier (la Révolution française) ${ }^{11}$.

\footnotetext{
7. Il est rare que les politistes investissent des objets antérieurs à 1789. À notre connaissance, le choix d'Y. Schemeil d'analyser la politique dans l'Ancien Orient afin d'y repérer des formes prémodernes d'organisation politique est un cas très isolé (Schemeil (Y.), La politique dans l'Ancien Orient, Paris, Presses de Sciences Po, 1999). La réception extrêmement critique de ce livre par les spécialistes a sans doute prémuni bien des politistes de s'aventurer en périodes inconnues.

8. D’où ce savoureux commentaire de Michel Offerlé décrivant la science politique comme un « jumard disciplinaire » (Offerlé (M.), « Haires et errances disciplinaires », in Déloye (Y.), Voutat (B.), dir., Faire de la science politique. Pour une analyse socio-historique du politique, Paris, Belin, 2002, p. 257).

9. Noiriel (G.), Introduction à la socio-histoire, Paris, La Découverte, 2008 ; Buton (F.), Mariot (N.), Pratiques et méthodes de la socio-histoire, Paris, Presses universitaires de France, 2009.

10. Participations, «La participation au prisme de l'histoire », 3, 2012.

11. Politix, «Protagonisme et crises politiques», 112, 2015. Pour une démarche similaire, cf. aussi Sewell (W. H. Jr), Logics of History. Social History and Social Transformation, Chicago, The University of Chicago Press, 2005.
} 
Notre propos ici est de prolonger ce type de coopération disciplinaire, en dépassant le seul échange de produits scientifiques finis (concepts, lectures ou méthodes). Notre travail a consisté, en amont, en des échanges collectifs réguliers et à modeler la construction théorique commune des hypothèses et du lexique scientifique ${ }^{12}$. L'enjeu était de trouver un angle d'approche sur les politisations ordinaires qui satisfasse tant l'historien.ne que le ou la politiste, c'està-dire qui permette des déplacements internes à chaque discipline (tout en les respectant chacune) et qui ne devraient qu'à la plus-value qu'autorise le travail interdisciplinaire.

\section{La science politique et I'histoire face aux politisations ordinaires}

Mettre en œuvre cette collaboration entre les deux disciplines implique le recours à des traditions historiographiques qui n'ont pourtant pas connu la même évolution. Car au cours des vingt dernières années, la science politique et l'histoire n'ont pas fait les mêmes usages, ni conféré la même place, à ce que nous appelons ici des politisations ordinaires. Si on peut parler, à partir des années 2000, d’un véritable agenda de recherche constitué, dans la sociologie politique française, autour de la question des formes ordinaires de rapport à la politique $^{13}$, il est plus difficile d'en dire autant en histoire.

En science politique, jusqu'aux années 1990, la discipline a consacrél'essentiel de ses efforts à l'analyse de l'espace social spécifiquement dédié aux questions politiques, de ses acteurs et de ses logiques : étude de la professionnalisation des activités politiques, saisie diachronique des cursus politiques (logiques de sélection du personnel, entretien dans le temps des positions politiques, etc.), intérêt pour les formes les plus institutionnalisées des produits politiques (comme les politiques publiques) ou pour les moments les plus saillants du fonctionnement des espaces politiques (comme les élections, les changements de régime, les situations de crises). Les citoyens ont pendant longtemps été rabattus sur les catégories de l'électeur, du sondé ou du militant, et surtout placés en situation de relative passivité par rapport aux diverses transactions que partagent les professionnels de la politique ${ }^{14}$.

Depuis une vingtaine d'années, l'accent a été davantage placé sur la porosité de ce champ politique à son environnement social (ce qui est sans doute la

12. Un séminaire organisé en 2015-2017, dans le cadre du projet « Politisations ordinaires » financé par le Labex SMS, a rendu possible la constitution d'un espace d'expérimentation entre historien.ne.s et politistes, dans lequel ont été forgés collectivement les concepts, hypothèses et lexiques dont rend compte ce numéro de Politix.

13. Cf. à ce sujet la contribution de Julien Weisbein à ce dossier.

14. Gaxie (D.), La démocratie représentative, Paris, Montchrestien, 1994. 
condition de son maintien ${ }^{15}$ ), empruntant à des grammaires qui lui sont extérieures (tout comme il les influence en retour), ainsi que diffracté dans d'autres univers sociaux (et pas seulement sur le mode du mimétisme) ${ }^{16}$. Les "profanes" se voient donc accorder de plus en plus de capacités et d'autonomie, notamment grâce à l'analyse de leurs représentations et comportements politiques. Leur politisation n'est plus saisie à partir d'une logique scolaire (consistant à reprendre plus ou moins fidèlement les biens politiques tels qu'ils sont légitimement consacrés) ${ }^{17}$, mais bien de manière interactionnelle, c'est-à-dire en contextes ${ }^{18}$, à partir d'ethnométhodologies, d'expériences ou de conceptions morales qui leur sont propres ${ }^{19}$ ou bien selon des raccourcis d'informations empruntés à leur vécu ${ }^{20}$. Des objets des plus classiques, comme l'explication du vote, ont été ainsi renouvelés par cet intérêt accru pour les facteurs extrapolitiques du politique ${ }^{21}$. Et c'est notamment dans une séquence qui s'ouvre dans les années 2010 qu'apparaît de plus en plus le mot «ordinaire » dans de nombreux travaux de politistes caractérisés par l'ambition de saisir des formes de raisonnement et de comportement politiques dans une granularité plus fine ${ }^{22}$.

15. Darras (É.), dir., La politique ailleurs, Paris, Presses universitaires de France/CURAPP, 1998 ; Le Gall (L.), Offerlé (M.), Ploux (F.), dir., La politique sans en avoir l'air. Aspects de la politique informelle, XIX ${ }^{e}$-XXI ${ }^{e}$ siècle, Rennes, Presses universitaires de Rennes, 2012.

16. Lagroye (J.), La politisation, op. cit.; Arnaud (L.), Guionnet (C.), dir., Les frontières du politique. Enquête sur les processus de politisation et de dépolitisation, Rennes, Presses universitaires de Rennes, 2005.

17. Blondiaux (L.), « Faut-il se débarrasser de la notion de compétence politique ? Retour critique sur un concept classique de la science politique », Revue française de science politique, 57(6), 2007.

18. Duchesne (S.), Haegel (F.), «La politisation des discussions, au croisement des logiques de spécialisation et de conflictualisation ", Revue française de science politique, 54(6), 2004 ; Hamidi (C.), "Éléments pour une approche interactionniste de la politisation. Engagement associatif et rapport au politique dans des associations locales issues de l'immigration ", Revue française de science politique, 56(1), 2006 ; Talpin (J.), "Ces moments qui façonnent les hommes. Éléments pour une approche pragmatiste de la compétence civique ", Revue française de science politique, 60(1), 2010 ; Eliasoph (N.), L'évitement du politique. Comment les Américains produisent l'apathie dans la vie quotidienne, Paris, Economica, 2010.

19. Berger (M.), «Répondre en citoyen ordinaire. Pour une étude ethnographique des engagements profanes ", Tracés, 15, 2008 ; Berger (M.), Cefaï (D.), Gayet-Viaud (C.), dir., Du civil au politique. Ethnographies du vivre-ensemble, Bruxelles, Peter Lang, 2011 ; Zambiras (A.), « Les sens de l'humour. Enquête sur les rapports ordinaires au politique », Politix, 96, 2011 ; Frère (B.), Jacquemain (M.), dir., Résister au quotidien?, Paris, Presses de Sciences Po, 2013.

20. Revue française de science politique, «Dimensions de la socialisation politique », 52(2), 2002 ; Aldrin (P.), "S'accommoder du politique. Économie et pratiques de l'information politique ", Politix, 64, 2003 ; Revue française de science politique, «La compétence politique. Nouveaux questionnements et nouvelles perspectives », 57(6), 2007.

21. Braconnier (C.), Dormagen (J.Y.), La démocratie de l'abstention. Aux origines de la démobilisation électorale en milieux populaires, Paris, Gallimard, 2007; Braconnier (C.), Une autre sociologie du vote. Les électeurs dans leur contexte : bilan critique et perspectives, Cergy-Pontoise, LEJEP-Lextenso Éditions, 2010 ; Lehingue (P.), Le vote. Approche sociologique de l'institution et des comportements électoraux, Paris, La Découverte, 2011 ; SPEL, Les sens du vote. Une enquête sociologique (France, 2011-2014), Rennes, Presses universitaires de Rennes, 2016.

22. Marie (J.-L.), Dujardin (P.), Balme (R.), dir., L'ordinaire..., op. cit.; Gautier (C.), Laugier (S.), dir., L'ordinaire et le politique, Paris, Presses universitaires de France/CURAPP, 2006; Lefébure (P.), « Les rapports ordinaires à la politique ", in Cohen (A.), Lacroix (B.), Riutort (P.), dir., Nouveau manuel de science politique, Paris, La Découverte, 2009; Bonny (Y.), Le Caro (Y.), Keerle (R.), Olitrault (S.), dir., Espaces de vie, espaces enjeux : entre investissement ordinaire et mobilisations politiques, Rennes, Presses universitaires 
Il est plus difficile d'identifier un «agenda de recherche » équivalent en histoire, car la discipline manque sans doute de concepts forts et partagés pour qualifier et circonscrire les rapports ordinaires au politique. Si l'on s'en tient à la catégorie de politisation - envisagée comme un processus vers la politique ou comme la participation politique d'acteurs habituellement écartés de ce domaine -, son emploi reste très limité dans les travaux portant sur les périodes antérieures au XIX ${ }^{\mathrm{e}}$ siècle, alors qu'il est devenu, pour les historien.ne.s de la période contemporaine, un outil conceptuel usuel ${ }^{23}$. De ce point de vue, l'histoire contemporaine récente dialogue bien plus facilement avec la science politique qu'avec l'histoire médiévale et moderne.

Pour ces deux dernières périodes, quelques tendances communes se dégagent lorsqu'il s'agit d'envisager les rapports ordinaires au politique. De nombreux travaux ont été récemment consacrés aux délibérations et assemblées populaires, aux agents subalternes, aux écritures ordinaires, ou aux rituels politiques impliquant les gens ordinaires, même si les éclairages restent limités à des objets circonscrits, qui empêchent parfois d'envisager l'espace politique dans son ensemble, et surtout les effets politiques de plus grande ampleur qu'ont ces acteurs et leurs actions sur la société ${ }^{4}$. Pour les historien.ne.s, le politique reste souvent considéré comme synonyme de pouvoir, de domination, d'État ou de gouvernement, entraînant une exclusion tacite des pratiques mises en ouvre par ceux qui ne sont pas dépositaires de l'autorité. La sphère politique est définie par la spécialisation liée à l'exercice du pouvoir, et dans la poursuite de ce raisonnement, envisagée d'abord dans sa dimension conflictuelle. L'obtention du pouvoir implique la lutte pour celui-ci, et la politisation repose sur le conflit ${ }^{25}$. Les gens ordinaires apparaissent dès lors dans ce tableau parce qu'ils sont considérés comme de potentiels destructeurs de l'ordre politique, comme si la politisation ne passait que par la révolte ou l'émeute, par la contestation

de Rennes, 2012 ; Carrel (M.), Neveu (C.), dir., Citoyennetés ordinaires. Pour une approche renouvelée des pratiques citoyennes, Paris, Karthala, 2014 ; Baamara (L.), Floderer (C.), Poirier (M.), dir., Faire campagne ici et ailleurs. Mobilisations électorales et pratiques politiques ordinaires, Paris, Karthala, 2016 ; Buton (F.), Lehingue (P.), Mariot (N.), Rozier (S.), dir., L’Ordinaire du Politique. Enquêtes sur les rapports profanes au politique, Lille, Presses universitaires du Septentrion, 2016.

23. Les enjeux sont bien résumés dans Fureix (E.), Jarrige (F.), La modernité désenchantée. Relire l'histoire du XIX siècle français, Paris, La Découverte, 2014, p. 233-234.

24. Au sujet de la délibération et du vote, cf. récemment Le Gall (L.), A voté. Une histoire de l'élection, Paris, Anamosa, 2017 ; Christin (O.), Vox Populi. Une histoire du vote avant le suffrage universel, Paris, Seuil, 2014 ; Otchakovsky-Laurens (F.), «S'assembler, délibérer, enregistrer au XIVe siècle : quand Marseille se constitue en institution ", Mélanges École française de Rome - Moyen Âge, 127-1, 2015. Au sujet des agents subalternes, cf. Dolan (C.), dir., Entre justice et justiciables. Les auxiliaires de la justice du Moyen Âge au XXe siècle, Québec, Les Presses de l'Université de Laval, 2005 ; Offenstadt (N.), En place publique. Jean de Gascogne, crieur au $X V^{e}$ siècle, Paris, Stock, 2013. Sur les rituels, Zemon Davis (N.), "The Rites of Violence », Society and Culture in Early Modern France, Stanford, Stanford University Press, 1975 ; Zemon Davis (N.), « Writing "The Rites of Violence" and Afterward », Past and Present, Supplement 7, 2012.

25. Bourquin (L.), Hamon (Ph.), dir., La politisation: conflits et construction du politique depuis le Moyen Âge, Rennes, Presses universitaires de Rennes, 2010. 
du pouvoir ou la lutte pour s'en emparer ${ }^{26}$. Même dans une perspective plus pacifique, c'est encore par le biais de la résistance à l'ordre que la politisation est envisagée ${ }^{27}$. Ce n'est que récemment que des travaux ont cherché à explorer, pour la période antérieure au XIX ${ }^{e}$ siècle, les formes et supports d'une popular politics $^{28}$, voire la question de l'existence d'un espace public au sens habermas$\operatorname{sien}^{29}$, ou plus généralement la participation et l'intervention des gens ordinaires dans le politique, en tentant de dépasser l'association systématique entre action et contestation politiques ${ }^{30}$.

\section{Trois approches de I'ordinaire}

À la suite de ces différents travaux, nous proposons de parler de "politisations ordinaires » afin d'éviter l'écueil des terminologies relationnelles ou en creux, qui trouveraient leur justification dans le rapport à un champ politique constitué, par imitation, par défaut ou dans la critique. Nous avons fait ainsi le choix d'évacuer les métaphores qui situent la politisation ordinaire dans sa subalternité ("populaire ", "par le bas », « au ras du sol », "à la périphérie », " ailleurs », " autochtone », "indigène ») ou dans une carence de légitimité ( « infrapolitique ", " informelle», " vernaculaire», " accidentelle», " autrement ", «profane », "non conventionnelle», « non élitiste», «sans en avoir l'air $»)^{31}$.

Le syntagme "ordinaire» (du latin ordinarius, ce qui est rangé par ordre) oscille souvent entre une acception médiane (l'homme ordinaire comme banal, sans qualité particulière ni traits distinctifs) et une acception désignant le point

\footnotetext{
26. L'article d'Edward P. Thompson est de ce point de vue fondateur: Thompson (E. P.), «The Moral Economy of the English Crowd in the Eighteenth Century ", Past and Present, 50, 1975 ; Tilly (Ch.), La France conteste de 1600 à nos jours, Paris, Fayard, 1986. Au sujet des révoltes médiévales, cf. les synthèses récentes Cohn (S.), Lust for Liberty. The Politics of Social Revolt in Medieval Europe, 1200-1425, Italy, France, and Flanders, Cambridge Mass., Harvard University Press, 2006 ; Firnhaber-Baker (J.), Schoenaers (D.), The Routledge History Handbook of Medieval Revolt, Londres, Routledge, 2017.

27. Évidemment, Scott (J.), La domination, op. cit. Récemment, cf. Hermant (H.), dir., Le Pouvoir contourné. Infléchir et subvertir l'autorité à l'âge moderne, Paris, Classiques Garnier, 2016.

28. Wood (A.), Riot, Rebellion and Popular Politics in Early Modern England, Basingstoke, Palgrave, 2002 ; Walter (J.), Crowds and Popular Politics in Early Modern England, Manchester, Manchester University Press, 2006 ; Rollison (D.), A Commonwealth of the People. Popular Politics and England's Long Social Revolution, 1066-1649, Cambridge, Cambridge University Press, 2010.

29. Boucheron (P.), Offenstadt (N.), dir., L'espace public au Moyen Âge: débats autour de Jürgen Habermas, Paris, Presses universitaires de France, 2011 ; Rospocher (M.), dir., Beyond the Public Sphere: Opinions, Publics, Spaces in Early Modern Europe (XVI-XVIII), Bologne, Il Mulino/Duncker \& Humbolt, 2012.

30. Harris (T.), The Politics of the Excluded, c. 1500-1850, Basingstoke, New York, Palgrave, 2001 ; Foronda (F.), dir., Avant le contrat social. Le contrat politique dans l'Occident médiéval (XIII ${ }^{e}$ XV siècles), Paris, Publications de la Sorbonne, 2011 ; Judde de Larivière (C.), La révolte des boules de neige. Murano face à Venise (1511), Paris, Fayard, 2014. Signalons, pour des temps plus anciens, Azoulay (V.), « Repenser le politique en Grèce ancienne ", Annales HSS, 3, 2014.

31. Michel Offerlé et Laurent Le Gall parlent au sujet de ces entreprises savantes de qualification d'un «étiquetage graphomane » (Offerlé (M.), Le Gall (L.), « La politique informelle entre incertitudes et inconstances », in Le Gall (L.), Offerlé (M.), Ploux (F.), dir., La politique sans en avoir l'air, op. cit., p. 9).
}

119 | politix 
bas d'une échelle de grandeur (l'ordinaire comme relevant d'une extraction basse) ${ }^{32}$. De fait, dans la littérature, la catégorie de l'ordinaire (la plupart du temps écrite entre guillemets) désigne bien souvent des réalités hétérogènes. Dans ce dossier, nous l'emploierons pour qualifier aussi bien des labels attachés aux personnes, des situations que des rapports sociaux.

En premier lieu, l'ordinaire peut donc être pensé comme un état ou un statut attribué à une personne ${ }^{33}$. Dans de nombreux travaux de science politique, on peut en repérer plusieurs variantes qui toutes renvoient au miroir de la domination et qui activent des formes de "politisation pratique ${ }^{34}$. Et dans cette optique, l'ordinaire du politique relève surtout de l'acquiescement à la domination $^{35}$, jusqu'à des petites tactiques invisibles de contestation ${ }^{36}$. Il s'agit d'abord d'un statut négatif qui consiste à ne pas être spécialisé en politique, mais à être profane, c'est-à-dire à être condamné à un éloignement statutaire par rapport aux transactions politiques les plus effectives ${ }^{37}$. L'ordinaire peut également renvoyer à l'anonymat ${ }^{38}$, un statut caractérisé par l'absence du support que constitue la classe sociale d'où on projetterait des traits typiques sur les individus afin de les caractériser ou de les organiser ${ }^{39}$, mais également par des positions

32. Dujardin (P.), «L’ordinaire démocratique: invention hiérarchique, impossible hiérarchisation ou le temps de l'homo eclecticus », in Marie (J.-L.), Dujardin (P.), Balme (R.), dir., L'ordinaire..., op. cit., p. 149.

33. Éric Chauvier parlerait à ce sujet de « désinterlocution » consistant à nier le statut d'interlocuteur aux enquêté.e.s pour les projeter dans l'espace théorique écrit maîtrisé par l'anthropologue (Chauvier (É.), Anthropologie de l'ordinaire. Une conversion du regard, Toulouse, Anarchasis, 2011).

34. La notion, esquissée pour rendre compte du rapport des ouvriers à la politique et des formes de dépossession par le Parti communiste, rend compte de ce que des événements parfois fortuits mais violents (brimades, vexations, dénis de droits, sentiments de gêne, etc.) peuvent induire de prise de conscience et de mobilisation contre une situation de domination (Pudal (B.), "Politisations ouvrières et communisme ", in Dreyfus (M.), Groppo (B.), Ingerflom (C.), Lew (R.), Pennetier (C.), Pudal (B.), Wolikow (S.), dir., Le siècle des communismes, Paris, Éditions de l'Atelier, 2000, p. 518).

35. Pour Nicolas Mariot d'ailleurs, l'objet d'une ethnographie de la citoyenneté consiste finalement à décrire les logiques de production du conformisme social (Mariot (N.), «Pourquoi il n'existe pas d'ethnographie de la citoyenneté ", Politix, 92, 2010, p. 193).

36. Scott (J.), La domination, op. cit.; Vairel (F.), Zaki (L.), « Politisation sous contrainte et politisation de la contrainte : outsiders politiques et outsiders de la ville au Maroc », Critique internationale, 50, 2011 ; Erdi Lelandais (G.), « Le quartier comme espace de résistance et de politisation. La Vallée de Dikmen à Ankara face à un projet de transformation urbaine », Cultures \& Conflits, 101, 2016.

37. Fromentin (T.), Wojcik (S.), dir., Le profane en politique. Compétences et engagements du citoyen, Paris, L’Harmattan, 2008 ; Aït-Aoudia (M.), Bennani-Chraïbi (M.), Contamin (J.-G.), «Indicateurs et vecteurs de la politisation des individus : les vertus heuristiques du croisement des regards ", Critique internationale, 50, 2011 ; Babeau (F.), «La participation politique des citoyens "ordinaires" sur l'Internet. La plateforme YouTube comme lieu d'observation ", Politiques de communication, 3, 2014 ; Buton (F.), Lehingue (P.), Mariot (N.), Rozier (S.), dir., L'Ordinaire du Politique, op. cit.

38. Ce que l'on retrouve également dans le champ éditorial, à travers certaines collections dédiées à la description de vies de « gens ordinaires » («Raconter la vie » au Seuil ou « Dans la peau de... » chez Armand Colin).

39. Deluermoz (Q.), Gobille (B.), « Protagonisme et crises politiques. Individus “ordinaires" et politisations "extraordinaires" ", Politix, 112, 2015, p. 14-15; Carrel (M.), Neveu (C.), "Pour un renouvellement des recherches sur la citoyenneté », in Carrel (M.), Neveu (C.), dir., Citoyennetés ordinaires, op. cit., p. 22. 
basses dans les hiérarchies militantes ${ }^{40}$. Cela va même dans de nombreux travaux jusqu'à l'équation "ordinaire égale populaire » ${ }^{41}$, avec ici la découverte que le désintérêt vis-à-vis de la politique institutionnelle constaté parmi les classes populaires n'interdit pas d'autres formes de politisation alimentées par des pratiques sociales liées au travail ou aux loisirs ${ }^{42}$. Localiser de la sorte la problématique de l'ordinaire en bas de l'échelle sociale est d'ailleurs souvent implicite dans les travaux des historien.ne.s, où l'expression " gens ordinaires » est fréquemment employée comme synonyme ou pour éviter celle de " gens du peuple ", de «classes populaires» ou de "gens d'en bas » ${ }^{43}$. Mais la catégorie demeure sans doute trop vague, les gens ordinaires restant le plus souvent définis en creux ou par défaut, comme ceux qui n’appartiennent pas à l'élite.

En second lieu, l'ordinaire peut qualifier une situation routinière, caractérisée notamment par l'absence d'événement politiquement significatif (élection, guerre, scandale, crise, émeute...), bref comme un temps froid, en dehors de l'exceptionnel, du hors-norme, de l'inédit. Ici, la catégorie renvoie tant au quotidien et à la routine qu'au permanent et à l'institué ${ }^{44}$. Elle permet surtout à la recherche de disjoindre les moments de politisation des citoyens des agendas politiques ou médiatiques, en s'intéressant aux routines de la vie quotidienne des premiers ou à des temporalités exclusivement biographiques ${ }^{45}$. L'analyse doit dès lors intégrer des éléments non discursifs ou non spécifiquement

40. Neveu (É.), «Trajectoires de soixante-huitards ordinaires », in Dammame (D.), Gobille (B.), Matonti (F.), Pudal (B.), dir., Mai-Juin 68, Paris, Éditions de l'Atelier, 2008 ; Bargel (L.), Jeunes socialistes/ jeunes UMP. Lieux et processus de socialisation politique, Paris, Dalloz, 2009 ; Pagis (J.), Mai 68. Un pavé dans leur histoire, Paris, Presses de Sciences Po, 2014 ; Joshua (F.), Anticapitalistes. Une sociologie historique de l'engagement, Paris, La Découverte, 2015.

41. Et de fait, il y a peu de travaux sur les rapports ordinaires au politique parmi les classes supérieures, à l'exception notable des travaux d'Éric Agrikoliansky ( La politisation ordinaire d'une population extraordinaire: les électeurs des "beaux quartiers" en campagne électorale (2006-2008) ", Politix, 106, 2014 ; "Logiques de l'oblique. Les rapports ordinaires au politique des "bourgeois" des beaux quartiers parisiens ", in Buton (F.), Lehingue (P.), Mariot (N.), Rozier (S.), dir., L'Ordinaire du Politique, op. cit.).

42. Hamidi (C.), «Éléments pour une approche interactionniste de la politisation », art. cit. ; Carrel (M.), «Politisation et publicisation : les effets fragiles de la délibération en milieu populaire », Politix, 75, 2006 ; Mischi (J.), «Les militants ouvriers de la chasse. Éléments sur le rapport à la politique des classes populaires », Politix, 83, 2008 ; Cossart (P.), Talpin (J.), « Les Maisons du Peuple comme espaces de politisation. Étude de la coopérative ouvrière la paix à Roubaix (1885-1914) ", Revue française de science politique, 62(4), 2012.

43. Te Brake (W.), Shaping History. Ordinary people in European Politics 1500-1700, Berkeley, University of California Press, 1998 ; Jacob (M. C.), Secretan (C.), dir., In Praise of Ordinary People. Early Modern Britain and the Dutch Republic, New York, Palgrave Macmillan, 2013.

44. Luc Boltanski parlerait lui du monde, défini comme «tout ce qui arrive ", comme «l'immanence même : ce en quoi chacun se trouve pris en tant qu'il est plongé dans le flux de la vie, mais sans nécessairement faire accéder au registre de la parole, encore moins de l'action délibérée, les expériences qui s'y enracinent» (Boltanski (L.), De la critique, Paris, Gallimard, 2009, p. 94). Ce monde s'oppose à la réalité, ce qui est construit socialement par les institutions dominantes - et qui renverrait dans notre dossier à la politique spécialisée.

45. Pagis (J.), «La politisation d'engagements religieux. Retour sur une matrice de l'engagement en mai 68 », Revue française de science politique, 60(1), 2010. 
politiques comme l'importance des corps, le divertissement, les émotions ou bien les appartenances communautaires ${ }^{46}$. Cette deuxième conception de l'ordinaire, qui doit beaucoup à Michel de Certeau ${ }^{47}$ ou à la phénoménologie, et qui en appelle essentiellement à des démarches ethnographiques ${ }^{48}$, est assez rare en science politique puisque le processus de politisation des citoyens s'apparente bien souvent à une réaction à des chocs exogènes, venant la plupart du temps du champ politique, ainsi qu'à des moments extraordinaires (dispositifs délibératifs, révolutions, attentats, etc.).

Rabattre l'ordinaire sur le temps banal de la vie quotidienne permet toutefois de poser une question d'apparence simple mais qui reste relativement peu traitée : se passe-t-il politiquement quelque chose quand il ne se passe rien? Ainsi en est-il par exemple de ces formes ordinaires de nationalisme, déconnectées des moments de mobilisation souvent rattachés à cette notion ${ }^{49}$, de la gestion des risques et des crises ${ }^{50}$ ou bien de ces formes de "résistance au quotidien » qui s'énoncent à des moments, dans des formats et sur des enjeux extérieurs à la politique institutionnelle ${ }^{51}$. Surtout, cette interrogation peut porter sur les espaces les plus institutionnalisés du politique. Avec la distinction qu'il pose entre l'ordinaire et le finalisé, Pierre Lefébure rappelle qu'il y a bien de l'ordinaire, du routinier ou de l'informel dans des univers politiques très institutionnalisés et spécialisés, notamment dans les moments où des produits politiques (investitures, votes, nominations à des postes, etc.) ne sont pas mis en jeu ${ }^{52}$.

46. Berger (M.), Cefaï (D.), Gayet-Viaud (C.), dir., Du civil au politique, op. cit.; Carrel (M.), Neveu (C.), dir., Citoyennetés ordinaires, op. cit.

47. De Certeau (M.), L'invention du quotidien. Tome 1. Arts de faire, Paris, Gallimard, 1980.

48. Céfaï (D.), «Vers une ethnographie du politique. Décrire des ordres d'interaction, analyser des situations sociales », in Berger (M.), Cefaï (D.), Gayet-Viaud (C.), dir., Du civil au politique, op. cit.; Participations, «Ethnographies de la participation », 4, 2012.

49. Billig (M.), Banal nationalism, Londres, Sage, 1995 ; Martigny (V.), « Penser le nationalisme ordinaire », Raisons politiques, 37, 2010.

50. Revet (S.), Langumier (J.), dir., Le gouvernement des catastrophes, Paris, Karthala, 2013 ; Becerra (S.), Lalanne (M.), Weisbein (J.), dir., Faire face aux risques dans les sociétés contemporaines, Toulouse, Octarès, 2016.

51. Frère (B.), Jacquemain (M.), dir., Résister au quotidien?, op. cit.; Breviglieri (M.), Gaudet (S.), «Les arrière-scènes participatives et le lien ordinaire au politique ", Lien social et Politiques, 71, 2014.

52. Lefébure (P.) "Les rapports ordinaires à la politique ", art. cit. C'est ce que montre par exemple très bien l'ouvrage de Guillaume Marrel et Laurent Godmer dans lequel l'ordinaire est synonyme à la fois de quotidien (de ce qui se passe dans le temps éveillé d'une élue) et de moment froid, en amont des périodes de préparation des élections. On y voit ainsi les « journées type » de cette élue, ce qu'elle fait « au jour le jour », cet angle mort des études sur les cursus politiques qui insistent plutôt sur les moments chauds. L'aspect heuristique de ce détour par le temps froid et le non spectaculaire consiste dès lors tant à qualifier des profils d'élu qu'à décrire plus finement le travail politique, et notamment la variété des tâches comme des publics qu'il induit (Godmer (L.), Marrel (G.), La politique au quotidien. L'agenda et l'emploi du temps d'une femme politique, Lyon, ENS Éditions, 2016). Le livre de Delphine Gardey sur l'Assemblée nationale est également riche de cette mixtion entre le noble et le trivial dans le travail de représentation politique attaché à l'institution parlementaire (Gardey (D.), Le linge du Palais-Bourbon. Corps, matérialité et genre du politique à l'ère démocratique, Lormont, Le Bord de l'eau, 2015). 
Ces situations ordinaires, telles qu'envisagées par M. de Certeau, ont donné des clés de lecture aux historien.ne.s pour penser le caractère routinier et quotidien de certaines pratiques ${ }^{53}$. Dans l'historiographie française, la mise à distance de l'événement par plusieurs générations de chercheurs a nécessairement contraint le débat sur la régularité et l'accident, faisant longtemps des situations routinières un impensé pourtant fondateur de la discipline. Récemment, c'est du côté des pratiques de l'écrit que la question de l'ordinaire est réapparue de façon explicite, à partir des usages quotidiens et routiniers des actes d'écriture et de la production d'une documentation qui renseigne sur le "presque rien » qui émerge parfois des sources ${ }^{54}$. De même, cette entrée par l'ordinaire permet une perspective déflationniste d'événements tels que les guerres ${ }^{55}$, comme elle amène à revisiter certaines institutions, à l'instar de la justice ${ }^{56}$.

En troisième lieu, dans une veine plus sociologique cette fois-ci, l'ordinaire peut renvoyer à un espace et à une échelle : le monde vécu, c'est-à-dire l'espace privé (le domestique, le matériel, l'alimentaire, le charnel, le loisir, l'intime, etc.) ou des espaces concrets (la rue, le quartier, le lieu de culte, la maison, etc. ${ }^{57}$. Dans cette définition plus scalaire de l'ordinaire, la science politique envisage le processus de politisation dans ses opérations de (re)cadrage, en insistant sur la manière dont les individus produisent une compréhension de leur univers direct, à travers l'expérience quotidienne qu'ils en font sur la base d'interactions répétées et dont les conversations peuvent à la fois recueillir des formes de politisation comme les infléchir ${ }^{58}$. Nicolas Mariot propose notamment une qualification de l'ordinaire par la densité des relations sociales et par l'importance des formes d'interconnaissance (ce qui s'oppose aux formes souvent déréalisantes, désingularisantes et universelles du politique comme la citoyenneté ou les idéologies partisanes) ${ }^{59}$. Dans cette approche, le processus de politisation n'est pas individuel mais induit par des collectifs divers dont la famille, les collègues au

53. Lüdtke (A.), Histoire du quotidien, Paris, Éditions de la MSH, 1994 ; Kott (S.), « De l'histoire sociale à l'Alltagsgeschichte. Entretien avec Alf Lüdtke », Genèses, 3, 1991.

54. Fabre (D.), dir., Écritures ordinaires, Paris, POL, 1993; Artières (Ph.), Rêves d'histoire. Pour une écriture de l'ordinaire, Paris, Les Prairies ordinaires, 2006; Bertrand (P.), Les écritures ordinaires. Sociologie d'un temps de révolution documentaire (entre royaume de France et Empire, 1250-1350), Paris, Publications de la Sorbonne, 2015 ; Stenckardt (A.), dir., Entre village et tranchées. L'écriture des poilus ordinaires, Uzès, Inclinaisons, 2015.

55. Agone, «L'ordinaire de la guerre », 53, 2014.

56. Piant (H.), Une Justice ordinaire. Justice civile et criminelle dans la prévôté royale de Vaucouleurs sous l'Ancien Régime, Rennes, Presses universitaires de Rennes, 2006 ; Argouse (A.), Je le dis pour mémoire. Testaments d'Indiens : lieux d'une justice ordinaire (Caja Marca, Pérou, XVII' siècle), Paris, Les Indes savantes, 2016.

57. Paperman (P.), «Versions sociologiques de l'ordinaire », in Gautier (C.), Laugier (S.), dir., L'ordinaire et le politique, op. cit.

58. Duchesne (S.), Haegel (F.), « La politisation des discussions, au croisement des logiques de spécialisation et de conflictualisation », art. cit. ; Eliasoph (N.), L'évitement du politique, op. cit. ; Braconnier (C.), « Le paradigme conversationnel dans l'analyse des rapports ordinaires au politique », in Buton (F.), Lehingue (P.), Mariot (N.), Rozier (S.), dir., L'Ordinaire du Politique, op. cit.

59. Mariot (N.), « Pourquoi il n'existe pas d'ethnographie de la citoyenneté », art. cit., p. 172. 
travail, les amis ou les voisins. On peut alors parler de « politisation par l'intime qui a pour cadre l'espace des relations interpersonnelles et privées " ${ }^{60}$, et de tous les espaces de liens ancrés dans la proximité (riveraineté, cercles de socialisation comme les associations ou les lieux de culte, etc. ${ }^{61}$. Mais l'ordinaire renvoie également à des espaces davantage publics comme les cercles professionnels, qui fournissent une base d'interconnaissance forte ainsi que des référents communs, et où l'on peut être amené à confronter son point de vue sur la politique avec des collègues d'opinions différentes ${ }^{62}$. Certains lieux (rue, quartiers, écoles, bidonvilles, églises, mosquées, plages, Maisons du peuple...) permettent également des processus de politisation en ce qu'ils peuvent être visés par une opération d'aménagement mais surtout parce qu'ils abritent et structurent des relations sociales souvent denses d'échanges, de loisirs, de solidarité ou d'identifications ${ }^{63}$.

Pour les historien.ne.s, cette question de l'échelle est depuis longtemps essentielle, et a impliqué différents débats. En regard des politisations, c'est bien entendu l'attention de Maurice Agulhon à la "République au village » qui a encouragé la prise en considération d'espaces plus limités et situés ${ }^{64}$. Les jeux d'échelle auxquels ont invité les micro-historien.ne.s italien.ne.s à partir des années 1980, ont réarticulé de façon dynamique les rôles politiques envisagés au ras du sol ${ }^{65}$. Le changement de focale et l'observation fine qu'ils autorisent jouent comme des révélateurs d'objets et de pratiques jusque-là rarement

60. Muxel (A.), «La politisation par l'intime. Parler politique avec ses proches », Revue française de science politique, 65(4), 2015, p. 544.

61. Hamidi (C.), «Éléments pour une approche interactionniste de la politisation ", art. cit.; "Catégorisations ethniques ordinaires et rapport au politique ", Revue française de science politique, 60(4), 2010 ; Zambiras (A.), «Les sens de l'humour », art. cit.; Carrel (M.), Neveu (C.), dir., Citoyennetés ordinaires, op. cit.

62. Biland (E.), «Les transformations générationnelles de la politisation dans les collectivités territoriales », Politix, 96(4), 2011 ; Pudal (R.), « La politique à la caserne. Approche ethnographique des rapports à la politique en milieu pompier », Revue française de science politique, 61(5), 2011 ; Sainsaulieu (Y.), Surdez (M.), Zufferey (E.), «La sociabilité politique entre travail et hors travail. Enquête sur la socialisation politique d'agriculteurs, d'ingénieurs et de directeurs des ressources humaines exerçant en Suisse ", in Buton (F.), Lehingue (P.), Mariot (N.), Rozier (S.), dir., L’Ordinaire du Politique, op. cit.

63. Hmed (C.), «Des mouvements sociaux "sur une tête d'épingle" ? Le rôle de l'espace physique dans le processus contestataire à partir de l'exemple des mobilisations dans les foyers de travailleurs migrants ", Politix, 84, 2008 ; Bonny (Y.), Le Caro (Y.), Keerle (R.), Olitrault (S.), dir., Espaces de vie, espaces enjeux, op. cit. ; Cossart (P.), Talpin (J.), «Les Maisons du Peuple comme espaces de politisation », art. cit.; De Blic (D.), Lafaye (C.), «Le travail politique du réseau Éducation sans frontières », in Frère (B.), Jacquemain (M.), dir., Résister au quotidien?, op. cit.; Aït-Aoudia (M.), «Des émeutes à une crise politique : les ressorts de la politisation des mobilisations en Algérie en 1988 », Politix, 112, 2015 ; Combes (H.), Garibay (D.), Camille Goirand (C.), dir., Les lieux de la colère. Occuper l'espace pour contester, de Madrid à Sanaa, Paris, Karthala, 2015 ; Norois. Environnement, aménagement, société, « Conflits de lieux, lieux de conflits », 238-239, 2016 ; Erdi Lelandais (G.), "Le quartier comme espace de résistance et de politisation », art. cit. ; Politix, "Mouvements d'occupation », 117, 2017.

64. Agulhon (M.), La République au village, Paris, Plon, 1970.

65. Revel(J.), dir., Jeux d'échelles. La micro-analyse à l'expérience, Paris, Gallimard/Seuil, 1996 ; Magnússon (S. G.), Szijártó (I. M.), What is Microhistory? Theory and Practice, Londres, Routledge, 2013. 
considérés. Selon une autre métaphore spatiale, c'est aussi "par le bas », from below, ou dans leur subalternité que certaines pratiques politiques ordinaires ont été mises au jour ${ }^{66}$.

\section{Saisir I'ordinaire}

Les articles de ce dossier renvoient à l'une, parfois à plusieurs, de ces trois caractérisations de l'ordinaire, avec toutefois la volonté collective d'en donner une définition positive, en ne le confondant ni avec la carence ou l'échec, ni avec le banal, mais en lui reconnaissant une forte valeur heuristique. À cette condition, la politisation peut être détachée du miroir grossissant que constituent les institutions politiques et l'État, sans nécessairement écarter ces dernières de l'analyse, mais sans leur conférer non plus une centralité ou l'exclusivité de la capacité d'action politique. Plutôt que de projeter par en haut des formes bien définies de ce qui est politique et ce qui ne l'est pas (formes et vocabulaire que, de surcroît, nous emprunterions a posteriori à la période contemporaine pour qualifier des configurations médiévales et modernes), nous préférons suivre l'invitation de la sociologie pragmatique en partant du sens vécu des acteurs, de leurs conceptualisations morales et de leurs capacités critiques, pour documenter d'autres états possibles du politique ${ }^{67}$. Dès lors, nous ne considérons pas ces formes ordinaires de politisation comme "faibles », limitées ou périphériques, mais nous nous intéresserons à des cas où elles sont effectivement centrales.

Il ne s'agit pas seulement de changer d'échelle ou de focale, mais de choisir le point de vue des acteurs en situation, des actions et des discours situés par lesquels ils agissent sur le monde auquel ils appartiennent. Pour comprendre ce que sont ces actions et ces discours, il faut donc interroger les ressources, les lieux d'apprentissage et les modalités d'émergence et de production du politique. Le quartier, le village, la communauté, la paroisse, le voisinage, l'atelier ou l'usine sont des lieux où sont créées, façonnées et perfectionnées des conceptions du commun et du monde. Par la spécificité de leur expérience de la vie collective, les hommes et les femmes " ordinaires » agissent et génèrent des discours spécifiques, qui sont à leur tour intégrés, digérés, assimilés par les élites, les gouvernants, la société et l'État. Ils contribuent ainsi à la production de catégories de gouvernement, d'institutions, de formes d'action, réflexives ou pas, qui façonnent profondément le monde et la société dans leur ensemble. Pour autant, il ne s'agit évidemment pas de poser un regard enchanté sur de

\footnotetext{
66. Voir les discussions récentes sur le forum «The Many-Headed Monster », [en ligne : http://manyheadedmonster.wordpress.com], en particulier le débat «Who is below? » engagé par Mark Hailwood. À ce sujet, Cerutti (S.), «Who is below? E. P. Thompson, historien des sociétés modernes : une relecture ", Annales HSS, 4, 2015. Spivak (G. C.), Les subalternes peuvent-elles parler?, Paris, Éditions Amsterdam, 2006 [1988]. 67. Boltanski (L.), L’amour et la justice comme compétences, Paris, Métailié, 1990 ; Barthe (Y.), de Blic (D.), Heurtin (J.-P.), Lagneau (É.), Lemieux (C.), Linhardt (D.), Moreau de Bellaing (C.), Rémy (C.), Trom (D.), «Sociologie pragmatique : mode d'emploi », Politix, 103, 2013.
} 
tels processus. Les politisations ordinaires peuvent être aussi conflictuelles que pacifiques, être le fruit ou l'objet d'une compétition parfois violente, et réengager à leur tour des situations de domination et de déséquilibre. Envisagées pour elles-mêmes, les politisations ordinaires donnent à voir un espace social complexe, qu'il faut saisir dans ces différentes dimensions.

L'analyse de ces formes de politisation renvoie donc à un problème d'ordre méthodologique et à la question des sources. Parce qu'elles sont ordinaires, ces formes sont souvent peu institutionnalisées, voire en gestation, dans un processus de formation qui ne s'est pas encore stabilisé. Pour les périodes anciennes, les politisations ordinaires s'appuient ainsi souvent sur des actions et des discours communautaires n'ayant pas laissé de traces écrites, soit parce qu'elles sont le fait d'hommes et de femmes ayant un accès limité à l'écrit, soit parce qu'il s'agit précisément de politisations reposant sur des usages oraux. Contrats, coutumes, accords tacites généraient des effets politiques, sans que nous ayons nécessairement accès aux débats et décisions les ayant produits. L'ordinaire de ces pratiques implique par définition qu'elles n'aient pas toujours d'échos dans les archives, car l'écrit est une forme d'institutionnalisation qui n'est pas nécessairement celle que nous cherchons. Pour les périodes plus récentes, le problème se pose également puisqu'il s'agit de saisir des actions et/ou des intentions avant qu'elles ne se formalisent, avant qu'elles ne s'institutionnalisent, avant même qu'elles n'adviennent, quand bien même elles se réaliseraient.

Pour autant, comme le démontrent les articles de ce dossier, les pratiques ordinaires ont laissé bien plus de traces qu'on ne pourrait le croire: dans les documents produits par les autorités d'abord, où les usages et pratiques communautaires sont enregistrés, organisés et encadrés par la loi, mais également dans de nombreuses autres sources qui ne sont pas celles traditionnellement considérées par l'histoire politique. Les archives des métiers, des paroisses ou des communautés, les sources judiciaires relevant du droit pénal comme du droit civil, les requêtes et les suppliques adressées au pouvoir, ainsi que certains documents privés sont pleins d'une rhétorique du politique et rendent compte de très nombreuses formes de politisation ordinaire qu'il s'agit d'observer en adoptant une focale adaptée.

\section{Dire et faire le commun}

À travers ces actions et ces mots, que défendent les acteurs et au nom de quoi agissent-ils ? Notre dernière hypothèse de travail a été que c'est dans l'énonciation de la possibilité d'un monde commun que se cristallisent ces politisations. Le commun, une notion récemment et relativement travaillée par les sciences sociales, ne sera pas considéré ici de manière essentialiste mais plutôt de façon indiciaire. Ce n'est pas de définir le commun qui nous intéresse, mais de comprendre comment sa mobilisation devient un principe et un moteur de l'action. 
On peut considérer que la science politique française a pour l'heure principalement appréhendé le commun dans l'optique de la théorie politique ou dans une approche économiciste des biens communs, c'est-à-dire des biens d'usages collectifs, ouverts, soustractibles et dont la régulation est problématique ${ }^{68}$. Cette caractérisation se retrouve notamment dans les travaux portant sur les politiques internationales liées à la protection de l'environnement, même si ceux-ci critiquent souvent une notion jugée trop proche des théories du choix rationnel et du néo-institutionnalisme libéral postulant une nécessaire coopération entre États pour le maintien de ces biens communs ${ }^{69}$. Dans les autres branches de la discipline, et notamment en sociologie politique ou en analyse de l'action publique, la référence au commun est d'ailleurs assez faible, sans doute en raison d'une volonté de démarcation vis-à-vis de l'économie et parce que la notion d'intérêt général est davantage mobilisée ${ }^{70}$.

En histoire, la question du commun cristallise de nombreux projets de recherche en cours, quelle que soit la période d'étude. Terme polysémique, le commun est d'une grande densité sémantique et constitue l'une des catégories essentielles de l'histoire politique des sociétés européennes. L'adjectif latin communis/e (qui appartient à tous ou est accessible à tous), comme le neutre commune/is (ce qui est en commun), ont constitué et généré des référents fondamentaux de la pensée et de la pratique politiques occidentales. Les sociétés médiévales se fondaient sur de nombreuses communautés, qu'elles soient rurales, villageoises, urbaines ou religieuses. À partir du XIe siècle, le monde des villes a été profondément renouvelé par les chartes de franchise à l'origine des Communes, constructions politiques contractuelles et communautaires originales ${ }^{71}$. Comme de nombreuses autres formations politiques médiévales, elles ont placé la défense du Bien commun à la base de leur projet politique, notion clé de la philosophie scolastique et des théories de gouvernement qui émergent avec Thomas d'Aquin ${ }^{72}$. Mais la terminologie

68. Ostrom (E.), La gouvernance des biens communs: pour une nouvelle approche des ressources naturelles, Bruxelles, De Boeck, 2010 [1990]. Les économistes poursuivent aujourd'hui cette réflexion, comme le montrent la participation de Jean Tirole au débat (Économie du bien commun, Paris, Presses universitaires de France, 2016) ou le récent Cornu (M.), Orsi (F.), Rochfeld (J.), dir., Dictionnaire des biens communs, Paris, Presses universitaires de France, 2017. Voir aussi «Biens communs, beni comuni », Tracés. Revue de Sciences humaines, 2016.

69. Critique internationale, "Politique de la biosphère », 9, 2000 ; Smouts (M.-C.), Forêts tropicales, jungle internationale. Les revers d'une écopolitique mondiale, Paris, Presses de Sciences Po, 2001 ; Le Prestre (P.), Protection de l'environnement et relations internationales. Les défis de l'écopolitique mondiale, Paris, Armand Colin, 2005 ; Morin (J.-F.), Orsini (A.), Politique internationale de l'environnement, Paris, Presses de Sciences Po, 2015 ; Gemenne (F.), dir., L'Enjeu mondial. L'environnement, Paris, Presses de Sciences Po, 2015.

70. À l'exception de Lascoumes (P.), Le Bourhis (J.-P.), « Le bien commun comme construit territorial. Identités d'action et procédures ", Politix, 42, 1998.

71. Boucheron (P.), Menjot (D.), La ville médiévale, Paris, Seuil, 2011, p. 289-333.

72. Kempshall (M.), The Common Good in Late Medieval Political Thought, Oxford, Clarendon Press, 1999 ; Lecuppre-Desjardin (É.), Van Bruane (A. L.), dir., De bono communi. Discours et pratique du Bien commun dans les villes d'Europe (XIII'-XVI siècle), Turnhout, Brepols, 2010. Pour l'époque antique, Macé (A.), «Deux formes du commun en Grèce ancienne », Annales HSS, 3, 2014. 
renvoie également aux gens du commun ou commoners, ceux qui n'appartiennent pas à la noblesse; ou encore aux communaux ou biens communaux, ces biens collectifs qui n'appartiennent à personne et que les communautés ont cherché à défendre contre les seigneurs. C'est peut-être dans les îles Britanniques que ces commons ont représenté l'enjeu politique le plus vif, un espace politique construit sur la Common Law, et où ce qui devient la House of Commons est fondé au $\mathrm{XIV}^{\mathrm{e}}$ siècle ${ }^{73}$. Jusqu'au " communisme », les déclinaisons de la catégorie sont, on le voit, d'une immense richesse, et attendent encore une synthèse historiographique ainsi qu'une analyse diachronique des transformations du concept.

Reste donc à comprendre ce que le commun représentait pour les gens ordinaires, comme valeur et justification de l'action, de façon à repérer s'il y avait un sens commun du commun. Notre objectif n'est pas d'analyser ce que désigne ce commun que les acteurs mobilisent dans leurs discours et leurs actions, mais plutôt comment celui-ci constitue un principe qui motive l'action. Saisir comment les gens disent et font le commun, dans des terrains situés et historicisés, revient à envisager leur mode de participation à la cohésion du monde social, observer comment ils en produisent le sens, en co-agissant, en disant et qualifiant le monde, en produisant des discours et des catégories morales. Les différents articles de ce dossier cherchent ainsi à circonscrire et à analyser des activités collectives, qui ont pour enjeu la construction d'un monde commun, et qui relèvent d'actions politiques ordinaires et quotidiennes.

Ces pratiques concrètes et discursives nous apparaissent comme porteuses d'un sens politique fort et comme relevant d'autant de pratiques politiques, justement par le fait qu'elles participent à la définition du monde commun et de ses règles. À la suite de P. Dardot et Ch. Laval, on peut considérer que le commun n'est pas seulement une affaire de gestion collective de certains biens ayant des propriétés intrinsèques nécessitant ce type de gestion, mais qu'il est avant tout un processus politique, une forme de co-activité, de praxis, caractérisée par la mise en œuvre de pratiques autonomes de discussion et de gestion collective de biens et de questions, par là construites comme devant échapper aux règles de l'appropriation privée - mais aussi de la gestion étatique ${ }^{74}$.

Cette conception du commun (constructiviste, en tant qu'elle ne donne pas de définition ontologique de ses frontières) nous semble entrer profondément en écho avec un ensemble de phénomènes que nous observons du XVI ${ }^{\mathrm{e}}$ au XIX siècle $^{75}$. Dans les différents terrains étudiés, il existe bien des pratiques

\footnotetext{
73. Watts (J.), «'Public or Plebs': The Changing Meaning of "the Commons", 1381-1549», in Pryce (H.), Watts (J.), dir., Power and Identity in the Middle Ages. Essays in Memory of Rees Davies, Oxford, Oxford University Press, 2007.

74. Dardot (P.), Laval (Ch.), Commun. Essai sur la révolution au XXI siècle, Paris, La Découverte, 2014.

75. Pour le $\mathrm{XX}^{\mathrm{e}}$ siècle et le contexte de l'extension de l'ordre marchand induit par la révolution néolibérale, cf. Lien social et Politiques, « La participation sociale et politique au quotidien », 71, 2014.
} 
de discussion et de gestion collective de ce qui touche l'ensemble d'un groupe ou d'une communauté. Elles ne sont pas pour autant réductibles aux règles de la négociation privée et à l'intervention directe de l'État ou du gouvernement, tout en entrant fréquemment en interaction avec les intérêts privés et avec l'action gouvernementale. S'ouvrent ici des lieux d'une politique, c'est-àdire d'un ensemble de comportements réglés, conflictuels parfois, mettant en jeu des relations de pouvoir, partiellement ouverts, et intégrés dans des processus qui touchent d'une manière ou d'une autre aux définitions des normes des conduites individuelles et collectives. Cette politique n'est pas celle de l'État, même si elle peut s'y mêler, être en partie produite par lui ou y conduire. Et elle trouve des formes d'inspiration dans des pratiques communautaires, religieuses, familiales, ouvrières, dont ce dossier analyse les mécanismes.

Comprendre cette politique incite à être attentif, enfin, à la possibilité de mondes qui ne sont pas encore là, et notre travail a également consisté à identifier les horizons d'attente qui motivent l'action, ces "futurs non advenus», craints ou espérés par des acteurs qui ne connaissent pas encore la fin de l'histoire $^{76}$. Ces "possibles du passé » nourrissent les actions et les discours que nous observons et, en situation, en constituent l'une des motivations fondamentales. En prenant au sérieux les idées et les idéaux que l'on peut saisir dans les sources, qu'ils soient de l'ordre du réel ou du possible, on peut reconstituer des formes non advenues, inaccomplies ou éphémères, mais qui sont autant d'alternatives et donc de mondes qui ont existé dans ces situations-là. Ces désirs et ces aspirations peuvent avoir à leur tour nourri des formes de discours et d'action reprises par les tenants du pouvoir, et c'est cette origine qu'il faut tâcher d'identifier. Notre objectif est donc d'intégrer à l'analyse ces mondes possibles qui constituent précisément des espaces de production d'un savoir et de conceptions politiques alternatives, car c'est aussi cela qu'engendrent les politisations ordinaires ${ }^{77}$.

C'est bien là, pour conclure, l'un des grands apports de l'analyse de ces formes politiques, qui permettent un autre récit de la modernité. La capacité du modèle de l'« État moderne » à s'imposer, et l'institutionnalisation, à partir du XIX $^{e}$ siècle, de formes canoniques et professionnalisées du politique, ont signifié la « défaite » de ces politisations ordinaires, et par conséquent leur progressive disparition du récit historique. La mise en forme historiographique a contribué à effacer ces expressions politiques alternatives du grand schéma narratif de la construction politique occidentale. Leur lente disparition, ou du moins leur soumission relative à des formes institutionnalisées d'action publique, a

76. Koselleck (R.), Le futur passé. Contribution à la sémantique des temps historiques, Paris, Éditions de l'EHESS, 1990 ; Deluermoz (Q.), Singaravélou (P.), Pour une histoire des possibles. Analyses contrefactuelles et futurs non advenus, Paris, Le Seuil, 2016.

77. Sur le modèle de ce que réalise Samuel Hayat avec l'idée de "représentation inclusive ", Quand la République était révolutionnaire. Citoyenneté et représentation en 1848, Paris, Seuil, 2014. 
pu laisser croire qu'il en avait toujours été ainsi. Mais en se replaçant dans le contexte potentiellement ouvert de l'Ancien Régime, et dans la perspective des possibles de l'époque, on perçoit l'importance de ce rééquilibrage analytique autant que narratif.

\section{Présentation du dossier}

Les cinq études de cas de ce dossier projettent ces questions et ces hypothèses dans une chronologie et une géographie larges, dans les villes et communautés rurales du Royaume de France pendant l'époque moderne et la Révolution, les villes et les villages de Navarre et les communautés villageoises d'Allemagne au XVIII siècle, ou enfin le bassin minier au nord de la France au XIX ${ }^{e}$ siècle. Ces cas sont saisis dans des moments de tension, mais chacun s'inscrit dans un temps long, avant, pendant et après que ne se manifeste le conflit, car ces formes de politisation ordinaire nourrissent une culture politique qui agit ensuite sur les interactions avec le pouvoir.

L'article de Jérémie Foa sur les pactes d'amitié produits durant les guerres de Religion montre la capacité des acteurs, à l'échelle locale, à s'accorder sur qu'ils ont en commun malgré leurs divisions religieuses, pour établir ce qui fonde un monde possible du vivre ensemble. En dépassant les divisions qui nourrissent la guerre civile, les habitants des villes et des villages participent ainsi à la construction d'une politique de la coexistence, par des pratiques locales et des arrangements, plutôt que des décisions politiques centralisées. Ils apparaissent ainsi bien loin de l'habituelle image de sujets passifs et obéissants, mais au contraire dans leur capacité collective à ressouder ensemble la communauté.

C'est une autre forme d'action politique locale qui occupe l'attention de Thomas Glesener, qui analyse de son côté un bien commun, à savoir le "porc des âmes", une pratique répandue dans l'Europe du Sud pendant l'Ancien Régime, qui consiste à nourrir collectivement un porc dont la vente financera des messes en l'honneur des morts. En repolitisant l'analyse de ce phénomène - à distance d'une approche purement religieuse ou folkloriste -, il s'agit de comprendre comment la question de la propriété d'un animal consacré aux âmes ouvre un espace de dissension qui met aux prises différents acteurs des communautés rurales (curés, magistrats, chefs de maison). Selon la qualification du porc comme un bien "sacré », " public » ou «commun », ce sont des définitions concurrentes de la localité, de son territoire et de l'appartenance à la communauté qui s'affirment.

Ce sont d'autres communautés et d'autres dépenses communes qui intéressent Rachel Renault, celles du Nord de l'Europe et du Saint-Empire romain germanique à la fin de l'Ancien Régime, qui, à plusieurs reprises, contestent collectivement la légitimité et l'utilisation de l'impôt. Or c'est un geste politique fort que de remettre en cause le principe organisant la fiscalité, et en analysant la 
conception que les acteurs mettaient en œuvre dans cette contestation, Rachel Renault analyse une forme ordinaire de politisation, non pas réduite à la protestation et au refus, mais au contraire inspirée d'une vision alternative de la défense du bien commun. C'est ainsi à rebours d'une interprétation canonique de la révolte comme destructrice de l'ordre qu'il faut au contraire chercher, dans le temps long de la construction de l'action politique, les fondements d'une aspiration politique autre, productrice d'une redéfinition du bien commun.

Les conflits qui émergent en Vendée au moment de la Révolution française à propos des nouveaux prêtres constitutionnels censés remplacer ceux refusant de prêter serment constituent l'observatoire choisi par Déborah Cohen pour analyser les discours politiques ordinaires articulés par des communautés. Les dénonciations et suppliques adressées au pouvoir par les habitants de ces villages pour défendre leurs prêtres, loin de révéler une opposition à la politique globale de l'Assemblée, témoignent d'une volonté collective de défendre la communauté, ses liens anciens et ses intérêts communs. Il y a donc deux niveaux de "politique », qui peuvent être ponctuellement en contradiction.

Enfin, c'est au sein de la Compagnie des Mines d'Anzin au XIXe siècle que Samuel Hayat cherche à saisir l'ordre alternatif tel que le construisent les mineurs, en produisant une définition du commun inspirée de leur organisation communautaire. Certes, la direction des mines cherche à les contrôler, mais cet ordre patronal n'est pas le seul à encadrer leurs pratiques. Cet exemple, à l'image de tant d'autres petites entreprises qui représentent le modèle dominant à l'époque, permet donc de comprendre comment se joue la définition du monde commun, par les acteurs eux-mêmes, dans les conflits les plus vifs tels que les grèves, mais aussi dans des formes plus ordinaires, par la construction d'un ordre communautaire qui définit une manière spécifique de dire et faire le commun, souvent opposée à celle des patrons. 
Claire JudDe DE LaRIvière est maître de conférences en histoire médiévale et de la Renaissance à I'Université de Toulouse/ Framespa (UMR 5136) et honorary research fellow au Birkbeck College, University of London. Elle étudie I'histoire de Venise entre la fin du Moyen Âge et la première modernité, en se concentrant sur la société vénitienne et les gens ordinaires: hiérarchies sociales, interactions, sociabilités, pratiques politiques ordinaires et relations avec la justice. Elle a notamment publié (avec Rosa Salzberg) " "Le peuple est la cité". L'idée de popolo et la condition des popolani à Venise (XVe-XVIe siècle)", Annales. Histoire Sciences Sociales, 2013/4, p. 1113-1140) et La révolte des boules de neige. Murano face à Venise, 1511 (Fayard, 2014).

judde@univ-tlse2.fr
Julien Weisbein est maître de conférences en science politique à Sciences Po Toulouse et chercheur au LaSSP (Laboratoire des sciences sociales du politique, EA4175). Ses travaux actuels portent sur les formes profanes de mobilisation face aux risques environnementaux, notamment sur les questions maritimes. II a récemment publié sur ce thème "Capter et (co)produire des savoirs sous contraintes : le tournant expert de Surfrider Foundation Europe ", Politix, 111 (3), 2015 ; « Défendre le littoral en croisant les expertises: le cas des Gardiens de la côte ", Norois. Environnement, aménagement, société, $n^{\circ} 238-239,2016 / 1-2$, p. 97-108. II a également co-dirigé avec Sylvia Becerra et Michèle Lalanne Faire face aux risques dans les sociétés contemporaines, postface de Claude Gilbert (Octarès, 2016).

julien.weisbein@sciencespo-toulouse.fr 